\title{
EL SUELDO DE LOS GRAMÁTICOS EN LA ANTIGÜEDAD ROMANA'
}

\author{
Urbano Espinosa \\ Universidad de La Rioja
}

\begin{abstract}
RESUMEN. Una inscripción romana del municipio romano Tritium Magallum (Tricio, La Rioja), dedicada a Lucius Memmius Probus, es el único documento epigráfico de la Antigüedad en el que se menciona el salario de un gramático. El presente estudio corrige la lectura dada tradicionalmente a la inscripción en lo que se refiere al sueldo del gramático tritiense, lo compara con los ingresos de otras profesiones y valora las iniciativas de los gobiernos locales para promover centros públicos de enseñanza.

ABSTRACT.A Roman inscription of the municipium Tritium Magallum (Tricio, La Rioja), deciicated to Lucius Memmius Probus, is the only epigraphical document of the Antiquity in which a grammarian's salary is mentioned. This study modifies the traditional reading given to the above-called inscription concerning the grammarian's salary, compares this one with the earnings related to some other jobs and values the initiatives of local governments to promote public teaching centres.
\end{abstract}

Son muy escasos los datos que ha legado el mundo romano sobre la situación económica de los docentes y en particular de los gramáticos, profesores que ocupaban el nivel intermedio en el esquema educativo de la Antigüedad. Por el contrario, son abundantes los datos disponibles sobre contenidos, objetivos y métodos en cualquiera de los tres grados por los que pasaba la formación completa de los jóvenes.

Sobre el objeto de nuestra pesquisa sólo disponemos de dos testimonios en las fuentes literarias. En primer lugar, sabemos que el liberto $M$. Verrio Flaco recibía 100.000 sestercios anuales como preceptor de los sobrinos de Augusto, si bien contaba con ingresos añadidos al poseer también una escuela privada ${ }^{2}$. En segundo lugar, el famoso gramático Q. Remio Palemón, maestro de Quintiliano, ganaba en su escuela particular 400.000 sestercios al año, al decir de Suetonio.3. Ambos gramáticos ejercieron en Roma y, si sus ingresos llegaron a merecer la atención de los

1. Adaptación del trabajo del autor, Das Gehalt eines grammaticus im westlichen Teil des Römischen Reiches: eine epigraphische Revision, Zeitschrift für Papyrologie und Epigraphik 68, 1987, 241-246

2. Suet., De gramm. 17.1-2

3. Ibid. 23.5; sobre otros ingresos complementarios, Plin. Nat. Hist. 14.49-50 
escritores de la época, fue por ser excepcionalmente elevados respecto a lo que debía ser habitual. Se trata, por consiguiente, de fuentes que no pueden ser tomadas en consideración como representativas de la situación económica media de los antiguos gramáticos.

La epigrafía mantiene un silencio total al respecto, con una sola excepción: una inscripción de la Hispania Citerior procedente del municipio Tritium Magallum (Tricio, La Rioja). Es el único testimonio epigráfico en el que se menciona el sueldo de un gramático latino. A pesar de su interés, la fuente ha permanecido escasamente atendida por la investigación actual sobre la educación en la Antigüedad romana ${ }^{4}$. Bien es cierto que a ello ha contribuido la inexactitud del texto transmitido hasta hoy. Fijar primero la correcta lectura del mismo y aportar luego una adecuada valoración histórica, constituyen el objeto del presente trabajo.

\section{La inscripción de Tritium Magallum}

El epígrafe que nos interesa fue descubierto en 1819 junto a otras doce inscripciones en las proximidades de la ermita de Nuestra Señora de Arcos, ubicada a unos 500 metros al Este de Tricio (La Rioja). Se trata de una inscripción funeraria dedicada al gramático latino Lucio Memio Probo, cuyas características formales no han sido transmitidas. En el momento del hallazgo el párroco de Tricio realizó una transcripción de todos los epígrafes descubiertos y remitió una copia a la Real Academia de la Historia (Madrid) ${ }^{5}$.

Desaparecido en una fecha indeterminada tras el hallazgo, ningún estudioso o investigador posterior ha podido ver el monumento original que ahora estudiamos. De ahí que aquella transcripción primera enviada a la Academia de la Historia constituya la única fuente disponible y de ahí también que las numerosas inexactitudes cometidas por el inexperto copista hayan llevado a diversos estudiosos a formular propuestas de recomposición de lo que podría ser el texto originario.

\section{a) El polémico texto de una inscripción desaparecida}

La copia del párroco de Tricio cayó en manos de A.C. de Govantes, quien en 1851 publicó por primera vez las trece inscripciones aparecidas simultáneamente, aunque sin que llegara a revisarlas en persona. Entre ellas, la del gramático Lucio Memmio Probo $^{6}$. De ese modo Govantes se constituyó en el intermediario imprescindible entre el documento manuscrito de la Academia de la Historia y la historiografía posterior. El texto que editó fue el siguiente:

4. Por ejemplo, es documento desconocido para H.-1. Marrou, Histoire de l'education dans l'Antiquité, Paris 1965 (6) y para S. F. Bonner, Education in Ancient Rome, Londres 1977.

5. J.A. Ceán Bermúdez, Sumario de las antigüedades romanas que hay en España, en especial las pertenecientes a las Bellas Artes, Madrid 1832, $189 \mathrm{~s}$.

6. A.C. de Govantes, Suplemento al Diccionario Geográfico-Histórico de la provincia de Logroño $y$ algunos pueblos de la de Burgos, Madrid 1851, 13, núm. 8. 


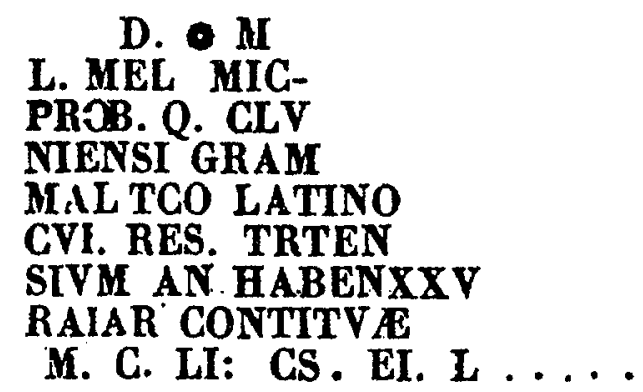

Emilio Hübner depende de A.C. de Govantes y lo mismo ocurre respecto a F. Baraibar, quien ya no pudo hallar la inscripción cuando en 1896 realizó una visita a Tricio"; Francisco Naval tampoco la encontró cuando la buscó en 1903 por encargo del Padre Fita. Hay que darla definitivamente por desaparecida.

El más serio intento de restituir el texto original es el realizado por Hübner, quien, tomando la versión de Govantes como referencia, modificó ostensiblemente su lectura. Sin embargo no resolvió, como veremos más adelante, la totalidad de los problemas. Reproducimos a continuación la propuesta de E. Hübner en CIL II 2892:

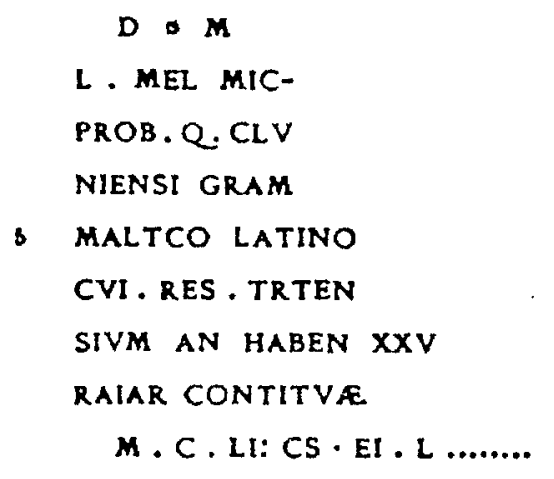

Baraibar y el resto de editores posteriores asumieron básicamente las correcciones de Hübneri; lo que quiere decir que, según la lectura de este último, el cluniense L. Memio Probo ganaría al año 1100 sestercios: [s]a[i]ar(ium) con[s]titu[it HS / MC (líneas 7 y 8 ). Es ahí donde discrepamos de la lectura dada por Hübner y proponemos un nuevo planteamiento.

7. E. Hübner, Act. Berolin. a. 1861, 948 (= CIL II 2892); de ahí, Diz. Epigr. (Ruggiero) III 565, con lectura incompleta; F. Baraibar, Lápidas romanas de Tricio, BRAH 50, 1907, $265 \mathrm{~s}$.

8. Han resultado infructuosos los intentos realizados por nuestra parte para hallar la copia original en la Real Academia de la Historia (Madrid); en todo caso, E. Hübner ya no la pudo hallar hacia mediados del s. XIX (CIL II p. 394: Tritium Magallum).

9. Con algunas modificaciones aceptan las correcciones de Hübner y Baraibar: L. Sagredo-S. Crespo, La enseñanza en la Hispania romana, Hisp. Antiqua 5, 1975, 124; J. C. Elorza et alii, Inscripciones romanas de La Rioja, Logroño 1980, núm. 48. 


\title{
b) Hacia una lectura correcta
}

Aceptamos como correcta la lectura de los editores anteriores desde la línea 1 hasta la 7 , pero consideramos que sigue siendo errónea la interpretación del texto precisamente en el punto en el que se alude al salario que percibía el gramático Lucio Memio Probo. Prestemos atención a la expresión contituae de la versión Govantes (final de lín. 8); no puede leerse con(s)tituit $\boldsymbol{H S}$ (o $n($ ummum) = sestercios), tal como hace Hübner, porque fuerza en exceso el cambio de la versión transmitida; con seguridad, el texto original hablaba de algo bien diferente. Según nuestra propuesta, la lín. 8 terminaba con(s)tit(uit) * (denarios), comenzando la lín. 9 con el numeral $\mathbf{M C}$. Veamos los argumentos en los que nos basamos.

La terminación VAE en Govantes era la lectura que el inexperto copista de 1819 propuso cuando vio al final de la línea 8 el signo * . Desconocía que se trata del indicativo de denarii y, por ello, lo interpretó como ligadura de las tres letras citadas; por lo demás, vio que las ligazones eran frecuentes en algunas de las otras 12 inscripciones descubiertas en Tricio en aquel momento. El error fue posible porque la parte superior del signo identificativo de denarios aparece como una $V$, la mitad inferior se asemeja a una $A$ y la línea horizontal configura una $E$ : VAE. No obstante, no hay que descartar del todo que el párroco local copiara correctamente la inscripción y que fuera Govantes quien en su edición de 1851 interpretara el signo * como ligadura de letras. En ese supuesto, diríamos que VAE era la única posibilidad de transcribir la paleografía romana con los medios de que disponía la tipografía en tiempos de Govantes.

Basándonos en todo ello, proponemos a continuación una lectura de la inscripción tritiense que, aceptando la versión de Hübner para las líneas 1 a 7 , reinterpreta, sin modificar apenas, la transcripción de Govantes en lo concerniente a la línea 8, justamente aquella cuya correcta lectura permite saber también con exactitud cuál fue el salario del gramático L. Memio Probo:

\author{
D(is) $\cdot$ M(anibus) \\ L(ucio) $\cdot \mathbf{M e}\lceil\mathbf{m}\rceil \mathbf{m i}\lceil\mathbf{o}\rceil$ \\ Prob $\lceil$ o 7 . Clu- \\ niensi gram- \\ 5 mal ti co Latino \\ cui res (publica) $\cdot \operatorname{Tr}[\mathrm{i}] \mathrm{t}[\mathrm{i}] \mathrm{en}$ - \\ sium an(nos) haben(ti) XXV \\ $\lceil\mathrm{s}\rceil \mathrm{a}\lceil\mathrm{l}] \operatorname{ar}$ (ium) con(s)tit(uit) $*$ ( $=$ denarios) \\ MC $\cdot\lceil h\rceil$ ic (est) s(it) $\cdot\lceil$ t(ibi) t(erra) $\rceil \cdot$ l(evis)
}

De esta lectura se deduce que Lucio Memio no recibía $1100 \mathrm{HS}$ (sestercios) de la res publica Tritiensium, como hasta hoy se había supuesto, sino que eran 1100 denarios $(=4400 \text { sestercios })^{10}$. Justamente un valor cuatro veces superior, con la consi-

10. Cfr. Al respecto, U. Espinosa, Epigrafia romana de La Rioja, Logroño 1986, núm. 25. 
guiente puesta en valor del papel social que correspondería a los gramáticos en la sociedad provincial romana.

La fórmula que encabeza el epígrafe - $D$ (is) M(anibus)- remite a una imprecisa cronología en el s. Il d.C. La desaparecida inscripción de Tricio es en sí misma un documento valiosísimo para los objetivos de este trabajo. Por ella sabemos que el municipio tritiense tutelaba y costeaba una escuela pública de gramática para los hijos de los conciudadanos; hay que suponer que sostendría otro centro para el primer nivel de la educación (los ludi literarii). Para regentarla contrató los servicios de un ciudadano de Clunia (Coruña del Conde, Burgos), capital del conventus iuridicus de su nombre en la Hispania Citerior. Lucio Memio Probo, hombre libre de nacimiento y ciudadano romano, contaba con 25 años de edad cuando recibió el encargo del municipio de Tritium y se trasladó a vivir a él, donde falleció a una edad que el documento no precisa. Su inscripción funeraria no menciona dedicante alguno, lo que probablemente significa que fue erigida y costeada por la propia institución municipal en prueba de reconocimiento por los servicios prestados.

El compromiso de Tritium a favor de una escuela de gramática se enmarca en el importante desarrollo económico alcanzado por la ciudad a través de su gremio de ceramistas. $Y$ es que por entonces Tritium Magallum era uno de los centros de Hispania más importantes en producción alfarera de terra sigillata ${ }^{11}$. En el s. II d.C. la expresión res publica Tritiensium, citada en el epígrafe, indica que la ciudad poseía la condición de municipio, privilegio que había recibido de alguno de los monarcas Flavios $^{12}$.

\section{La posición económica del gramático tritiense}

Para valorar el significado socioeconómico del salario anual percibido por L. Memmio comparémoslo brevemente con algunas escalas coetáneas de sueldos. En el s. II d.C. un trabajador normal ganaba de 1 a 4 HS por día; la ley de la colonia Urso (Osuna, Sevilla), cuyo texto actualmente conocido es de época flavia, fija las percepciones anuales de los funcionarios de la administración local como sigue: $300 \mathrm{HS}$ para el tibicen, para el librarius y para el praeco; 400 para el viator; 500 para el haruspex, 600 para el lictor; 700 para el accensus y $1200 \mathrm{HS}$ para el scriba ${ }^{13}$. El stipendium de un soldado en el s. II d.C. era de $1200 \mathrm{HS}$, de los cuales aproximadamente tres cuartas partes se le retenían para manutención, armamento y otras necesidades; ahora bien, si el legionario lograba el ascenso a los rangos superiores de tropa, entonces podía recibir de 1,5 a 3 veces más. En cuanto a los mandos del ejército, sólo recordaremos aquí el grado de centurión, cuyo nivel básico percibía $20.000 \mathrm{HS}$ anuales ${ }^{14}$.

11. T. Garabito, Los alfares romanos riojanos, Madrid 1978; F. Mayet, Les céramiques sigillées hispaniques, París 1984.

12. U. Espinosa y A. Pérez Rodríguez, Tritium Magallum: de ciudad peregrina a municipio romano, AEArq. 55, 1982, 75 ss.

13. Lex. Urs. 62; cfr. A. D'Ors, Epigr. Jurídica de la España romana, Madrid 1952, 179-182.

14. A. von Domaszewski, Der Truppensold des Kaiserzeit, en Neue Heidelb. Jahrb. 10, 1901, 230; G. R. Watson, The Roman soldier, Londres 1969, 91. 
Se acepta comúnmente que $250 / 300$ HS por año era el mínimo de recursos necesarios en el Alto Imperio para cubrir las necesidades más perentorias. Con otras palabras, aproximadamente los ingresos de un praeco en Urso o de un trabajador manual cualquiera, suponiendo que este último ganara un sestercio al día y estuviera ocupado la totalidad del año'.

Si retornamos ahora al gramático tritiense, observamos que su sueldo de $4.400 \mathrm{HS}$ multiplicaba casi por cuatro el de un escriba de Urso, el mejor remunerado de la administración local. La misma proporción se establece respecto a la soldada de un legionario, si bien cuando éste alcanzaba el rango de los principales entonces se acortaba notablemente la diferencia respecto al sueldo del docente de Tricio. Por el contrario, la paga básica de un centurión era unas cinco veces superior a la de L. Memio. Como consecuencia de lo anterior, podemos suponer con verosimilitud que el volumen de los ingresos anuales, permitía al gramático llevar un ritmo de vida acomodado, al menos en relación con un trabajador manual, con un legionario de la escala básica o con un funcionario inferior de la administración de un municipio o de una colonia. Tampoco daba para demasiados lujos.

\section{Unos docentes desigualmente remunerados}

Hasta aquí las equiparaciones que pueden obtenerse para ponderar el valor del sueldo de Lucio Memio en relación con algunas escalas de salarios de su propio tiempo. Más difícil resulta el intento de compararlo con el de gramáticos de otras localidades y provincias; sencillamente, porque apenas disponemos de una información mínima.

a) Éxito y pobreza de una profesión liberal

Al principio mencionamos que los ingresos de $M$. Verrio Flaco y de $Q$. Remio Palemón en Roma tenían carácter singular y no eran indicativos de niveles medios de la profesión' ${ }^{\prime}$. Como primer criterio, no hay que olvidar que la mayoría de los gramáticos de los que tenemos noticia son de origen servil ${ }^{17}$. Tras la manumisión el liberto podía abrir su propia escuela privada y a partir de ahí actuar como un hombre libre; este fue el caso típico de buen número de gramáticos.

Según Suetonio y Juvenal eran docentes mal remunerados, que cobraban muy irregularmente y que, no era raro, llevaban una vida llena de estrecheces, llegando a morir en la miseria ${ }^{18}$. Marrou opina que esta situación era la más frecuente ${ }^{19}$.

15. Th. Pekáry, Die Wirtschaft der griechisch-römischen Antike, Wiesbaden 1976, 109 y 111.

16. No incluimos aquí la noticia de los 2000 dracmas egipcios que Lollianus, el grammaticus de la escuela pública de Oxyrhynchus, ganaba hacia el 253-260 d.C., pues en ese momento la moneda ya no posee el mismo valor que en el s. II d.C. (P. J., Petitions and Letter: the grammarian's complaint, Collect. Papyrologica to H. C. Youtie, II, 1976, 409-446.

17. J. Christes, Sklaven und Freigelassene als Grammatiker und Philologen im antiken Rom, Wiesbaden 1979, refiriéndose a Roma e Italia, cuyas conclusiones, no obstante, son extrapolables a la situación de las provincias. Para la información epigráfica provincial, Cfr. Diz. Epigr. (Ruggiero) III $564 \mathrm{~s}$.

18. Según Suetonio, L. Orbilius se veía obligado a vivir en una buhardilla y había tenido una vejez miserable (De gramm. 9.1-2). Según Juvenal, (Sat. VII 228) conseguían el dinero de sus clientes de tarde en tarde (rara merces).

19. H.-1. Marrou, op. cit. 1965, 401 
En contraposición a lo anterior, no obstante, hay que señalar que la situación económica real podía mostrar fuertes contrastes según los casos. No hay que olvidar factores de diferenciación como la generosidad o tacañería de los padres de los alumnos, el prestigio profesional del profesor mismo, los recursos y la política educativa de la ciudad en la que se enseñara o la competencia que pudiera existir entre docentes. La disparidad de situaciones es, a nuestro entender, la característica más determinante de los gramáticos dedicados al libre ejercicio de la profesión, tanto de los nacidos ingenui como de los que eran liberti.

\section{b) Gramáticos e instituciones locales}

Nuestro gramático Lucio Memio no ejercía el oficio en Tritium por su propia cuenta. Hay que incluirlo entre aquellos docentes que estaban al servicio de las instituciones locales y que de ellas dependían para regentar centros de titularidad pública. Conocemos otros tres casos más de gramáticos relacionados con los gobiernos locales. Sabemos que en la ciudad de Como (Italia) el ordo Comensium otorgó los ornamenta decurionalia al gramático latino P. Atilio Septiciano ${ }^{20}$; desconocemos si enseñaba o no en la schola que Plinio fundó generosamente en esa ciudad ${ }^{21}$. Igual que en el caso anterior, también el gramático latino Q. Tuticano Eros fue honrado en Verona (Italia) con los ornamenta decurionalia ${ }^{22}$. En Benevento (Italia) era gramático de profesión el decurio M. Rutilio Eliano 23 .

En ninguno de estos tres testimonios se menciona si los gramáticos regentaron una escuela pública. En los casos de Como y Verona se dice que recibieron del senado local la distinción de decuriones honoríficos, previsiblemente en un momento avanzado de su vida. En el caso de Benevento, simplemente se afirma que un senador local se dedicaba a la enseñanza como gramático. No existe, pues, mención explícita a una eventual vinculación de los personajes con centros educativos costeados por su respectiva ciudad.

Sin embargo, dado que alcanzaron el rango de decuriones, cabe preguntarse a cuánto ascendían sus ingresos o cuál era su patrimonio en relación con las exigencias legales de poseer determinada renta para poder alcanzar el decurionado ${ }^{24}$. De los tres personajes citados, el beneventano $M$. Rutilio fue el único decurión efectivo y carecemos de datos para saber si alcanzaba el mínimo de renta con los ingresos profesionales o con el patrimonio privado y familiar. Q. Tuticano en Verona y P. Atilio en Como eran decuriones honorarios y probablemente no alcanzaban el mínimo de $100.000 \mathrm{HS}$ que se exigían para alcanzar el rango efectivo de decurión ${ }^{25}$. Hemos de suponer, no obstante, que la situación económica de los gramáticos elevados honoríficamente a dicho rango debía ser tal que no chocaría con la dignitas propia del honos decurionalis que recibieron.

20. CILV 5278 (= LLS 6729).

21. Plin. Epist. IV 13.

22. CIL $\vee 3433$.

23. CIL IX 1654.

24. J. Christes, Bildung und Gesellschaft, Darmstadt 1975, 241 s. Nota 333.

25. Precisamente Plinio menciona esa cifra a propósito de Como, su ciudad natal (Epist. I 19). 
La inscripción de Tritium Magallum aporta en este sentido cierta información. Teniendo en cuenta el salario de 4.400 HS que Lucio Memio percibía anualmente, debía ser altamente improbable que un gramático, con independencia de que regentara una escuela pública o privada, pudiera alcanzar las rentas exigidas para el decurionado sobre la base exclusiva de sus ingresos como docente.

En síntesis, conocemos cuatro gramáticos vinculados de uno u otro modo a las instituciones locales, pero Memio Probo sigue siendo el único caso del que con seguridad sabemos que regentaba una escuela pública. La falta de documentación no nos permite deducir, sin embargo, que fuera una excepción la figura de los gramáticos en los municipios y en las colonias del Imperio Romano. Al contrario; un decreto de Antonino Pío (aproximadamente de la misma época que L. Memio) otorgaba la inmunitas (exención de cargas) a un determinado número de médicos, sofistas y gramáticos por cada ciudad ${ }^{26}$. Esta legislación imperial, en sí misma, obliga a suponer que en el siglo II d.C. no eran raras las ciudades en las que podía cursarse el segundo grado de la educación. Por tanto, la relación de Memio Probo con las instituciones locales no debía ser un caso aislado en el panorama educativo; sólo constituye para nosotros una fuente aislada en un panorama general del que no nos ha llegado información.

\section{Tritium como ciudad de tipo medio}

Gracias a una abundante documentación epigráfica y arqueológica podemos trazar un bosquejo histórico bastante preciso sobre la realidad social y económica de Tritium en el s. Il d.C. Tal como indicamos arriba, la ciudad llegó a ser uno de los principales centros de producción alfarera del occidente latino, logrando unas cotas difícilmente imaginables de exportación de cerámica a toda la Península lbérica y al norte de África. En la actualidad conocemos más de doscientas estarnpillas o marcas distintas de productores de cerámica (officinatores), siendo previsible un notable incremento del inventario a medida que vayan avanzando las investigaciones. El producto tritiense más característico fue la llamada terra sigillata, cuyo floruit se dio desde el último cuarto del s. I d.C. hasta finales del s. II d.C.

La escuela de gramática regentada por Lucio Memio Probo encaja precisamente en ese periodo, y es que la riqueza acumulada en Tricio por el floreciente comercio alfarero fue la base de su importante desarrollo cultural y social. La ciudad había recibi-do el rango de municipio en las últimas décadas del s. I d.C., hecho que potención su actividad económica. Tricio contaba con un destacamento de la Legión VII Gémina permanentemente asentado en su solar, constituyendo un factor añadido que contribuía a la plena apertura de la zona a las corrientes de universalidad del momento. La ciudad puede mostrarse como un significativo ejemplo de implantación de las formas de vida a la romana, adaptando a ellas, sin provocar la desaparición, diversos elementos culturales de la ciudad berona que fue en origen.

26. Dig. 27.1.6.1 y 8 (Vespasiano y Adriano: Dig. 50.4.18.30). Tritium tendría el nivel de ciudad pequeña, y por tanto podía eximir de impuestos (inmunitas) como máximo a 5 médicos, a 3 sofistas y a 3 gramáticos. 
Conocemos diversas sagas familiares de alfareros tritienses, llegando alguna de ellas, como la de los Mamilios, a situar diversos miembros en los puestos más relevantes de la provincia Hispania Citerior. Buena muestra del poder económico de la ciudad y del desarrollo romanizador en su solar lo constituía el monumental templo erigido hacia el s. II d.C. Seguramente fue costeado por la hacienda municipal. Fustes y sillares del monumento fueron reutilizados hacia finales del s. IV ó s. V en la basílica paleocristiana de $\mathrm{N}^{\mathrm{a}}$ Sra. de Arcos (Tricio) y muestran una envergadura en sus proporciones superior a la inmensa mayoría de los vestigios similares conocidos a lo largo y ancho de Hispania.

Sirvan estos breves apuntes de carácter histórico y arqueológico para definir el clima en el que surgió la escuela tritiense de gramática y el entorno en el que Lucio Memio Probo desarrolló su labor. Hay que contar con que en el valle medio del Ebro al menos en Calagurris (Calahorra) hubiera algún centro de gramática, sin perjuicio de que pudieran existir otros en ciudades como Graccurris (Alfaro) o Turiaso (Tarazona). En todo caso, en los enclaves urbanos del Ebro, fuertemente romanizados, debió ser habitual disponer de profesores para el primero de los niveles educativos.

Hemos querido detenernos en estas referencias históricas para mostrar que Tritium en el siglo II d. C. no representaba un caso aislado, ni en el contexto más próximo del valle del Ebro ni en el conjunto del occidente latino. De ahí que concluimos este apartado afirmando que la ciudad alcanzó en la época mencionada un desarrollo económico, social y cuitural similar al de Como y Verona en Italia, en relación con los testimonios epigráficos a los que más arriba hemos aludido. Por ello, el caso de Lucio Memio puede servir como ejemplo de una situación más general (la colonia beneventana debía ser una ciudad mayor que las dos itálicas mencionadas).

\section{Vida municipal y enseñanza de la gramática}

Tritium Magallum representan bien el modelo de ciudad de tamaño medio consolidado en el occidente del Imperio Romano, pues eran excepción los grandes centros económicos y administrativos. Seguro que no todas las ciudades pagaban lo mismo a los gramáticos de sus respectivas escuelas públicas; dependía, entre otros factores, de las posibilidades financiera de cada hacienda local, del auge económico de la ciudad, así como del número de niños a educar.

Pero a diferencia de lo que ocurría con los gramáticos que ejercían libremente la docencia, es muy probable que quienes regentaban una escuela pública definieran un grupo de docentes con ciertas condiciones de homogeneidad y privilegio: no estaban expuestos a las inversiones y riesgos de un centro privado, ni tenían que competir con otros colegas para captar alumnos o para sobrevivir económicamente. Si no había quiebra de las finanzas locales, la caja de la ciudad les pagaría puntualmente sus honorarios. De ahí que en la inscripción de Tritium se hable de salarium ${ }^{27}$ y no de merces, a la que aludía Juvenal ${ }^{28}$, como sería el caso de los ingresos que percibían los docentes privados.

27. En el documento de Lollianus en Oxyrhynchus se utiliza el vocablo "syntaxis" como sinónimo de "misthos" (Cfr. Nota. 16).

28. Juvenal, Sat. VII 228; Suet. De gramm. 3. 


\section{Conclusión}

La legislación imperial respecto a la exención de cargas a los gramáticos prueba que éstos eran frecuentes en las ciudades de las provincias romanas, tanto si regentaban escuelas públicas como privadas. Por otro lado, hemos constatado que Tritium alcanzó en el s. II d.C. un nivel demográfico, económico y cultural equiparable al de cualquier otra ciudad de tamaño mediano en el occidente del Imperio Romano. Pero frente a esta relativa generalización de profesores en el grado intermedio del sistema educativo antiguo, sigue siendo una incógnita, dada la ausencia de información paralela, si el sueldo de L. Memmius Probus era o no equiparable al de otros responsables de escuelas públicas de gramática. Sea como fuere, no cabe duda que percibía por su trabajo un salario realmente notable. 